\title{
A microRNA-dependent program controls p53-independent survival and chemosensitivity in human and murine squamous cell carcinoma
}

\author{
Benjamin Ory, ${ }^{1}$ Matthew R. Ramsey, ${ }^{1}$ Catherine Wilson,, ${ }^{1}$ Douangsone D. Vadysirisack, ${ }^{1}$
} Nicole Forster, ${ }^{1}$ James W. Rocco, ${ }^{2}$ S. Michael Rothenberg, ${ }^{1}$ and Leif W. Ellisen ${ }^{1}$

${ }^{1}$ Massachusetts General Hospital Cancer Center and Harvard Medical School, Boston, Massachusetts, USA. ${ }^{2}$ Division of Surgical Oncology, Massachusetts General Hospital and Massachusetts Eye and Ear Infirmary, Boston, Massachusetts, USA.

\begin{abstract}
The p53 tumor suppressor, a central mediator of chemosensitivity in normal cells, is functionally inactivated in many human cancers. Therefore, a central challenge in human cancer therapy is the identification of pathways that control tumor cell survival and chemosensitivity in the absence of functional p53. The p53-related transcription factors $\mathrm{p} 63$ and $\mathrm{p} 73$ exhibit distinct functions $-\mathrm{p} 73$ mediates chemosensitivity while p63 promotes proliferation and cell survival - and are both overexpressed in squamous cell carcinomas (SCCs). However, how $\mathrm{p} 63$ and $\mathrm{p} 73$ interact functionally and govern the balance between prosurvival and proapoptotic programs in SCC remains elusive. Here, we identify a microRNA-dependent mechanism of $\mathrm{p} 63 / \mathrm{p} 73$ crosstalk that regulates p53-independent survival of both human and murine SCC. We first discovered that a subset of p63-regulated microRNAs target $\mathrm{p} 73$ for inhibition. One of these, miR-193a-5p, expression of which was repressed by $\mathrm{p} 63$, was activated by proapoptotic $\mathrm{p} 73$ isoforms in both normal cells and tumor cells in vivo. Chemotherapy caused $\mathrm{p} 63 / \mathrm{p} 73$-dependent induction of this microRNA, thereby limiting chemosensitivity due to microRNAmediated feedback inhibition of p73. Importantly, inhibiting miR-193a interrupted this feedback and thereby suppressed tumor cell viability and induced dramatic chemosensitivity both in vitro and in vivo. Thus, we have identified a direct, microRNA-dependent regulatory circuit mediating inducible chemoresistance, whose inhibition may provide a new therapeutic opportunity in p53-deficient tumors.
\end{abstract}

\section{Introduction}

The p53 family transcription factors, including p53 (TP53), p63 (TP63), and p73 (TP73), play key roles in development, tumorigenesis, and the response to DNA damage. The p53 tumor suppressor is a central mediator of the DNA damage response and chemosensitivity in normal cells, yet it is functionally inactivated by mutation or other mechanisms in the majority of human cancers (1). Therefore, intensive efforts in cancer therapeutics have focused on identifying an effective means to induce tumor-cell killing in the setting of inactivated p53. One such strategy involves the restoration of p53 function itself in tumor cells through a wide variety of interventions depending on the mechanism of p53 activation in a given tumor (2). Nevertheless, successfully treating the largest fraction of human cancers will require approaches targeting p53independent survival pathways.

While p53 is associated with growth arrest and is not required for normal embryonic development, p63 mediates regenerative proliferation and is required for development of ectoderm-derived tissues (3-5). In cancer, $\mathrm{p} 63$ has been linked to cellular survival, in keeping with genomic amplification and/or overexpression of $\mathrm{p} 63$ observed in a subset of tumors including squamous cell carcinoma (SCC) (6-8). Genetic models of p73 loss imply a p53-like role in promoting genomic stability $(9,10)$. Like p53, p73 also functions as an essential mediator of cell death in response to chemotherapy

Conflict of interest: The authors have declared that no conflict of interest exists. Citation for this article: J Clin Invest. 2011;121(2):809-820. doi:10.1172/JCI43897. and other forms of DNA damage, particularly in tumor cells with inactive p53 (11-13). Given that p73 is a potent inducer of apoptosis, endogenous cellular p73 levels are tightly controlled through both transcriptional and posttranslational mechanisms $(11,13)$. Nevertheless, a complete understanding of how p73 levels and activity are regulated is lacking.

Remarkable structural homology exists among p53 family members, particularly in their DNA binding and oligomerization domains $(14,15)$. Furthermore, both p63 and p73 exhibit expression from 2 distinct promoters, thereby producing protein isoforms that either contain or lack the $\mathrm{N}$-terminal transactivation domain (TA and $\Delta \mathrm{N}$ isoforms, respectively). These features have provoked intense interest in possible modes of functional interaction among family members. One such mechanism involves binding by multiple family members to shared DNA consensus motifs within regulatory regions of particular target genes (16). Additionally, since all p53 family transcription factors must oligomerize to bind DNA, hetero-oligomer formation between different family members is another potential mechanism of functional interaction, particularly between p 63 and p73 (17). Both shared promoter binding and direct physical interaction have been demonstrated between p63 and p73 in SCC, a tumor in which both p63 and p73 are known to be overexpressed $(18,19)$. Specifically, $\Delta \mathrm{Np} 63 \alpha$ has been shown to inhibit TAp73 function in SCC cells through direct binding to the promoters of TAp73 target genes and through direct physical association with TAp73 both in vitro and in vivo (8, 19). Treatment of SCC cells with DNA-damaging agents including 
cisplatin induces a p73-dependent cell death program as a result of both p73 activation and p63 degradation (8, 20-22). Although platinum-based chemotherapy remains a standard treatment approach for these tumors, SCC remains among the most treatment refractory of human cancer subtypes. Thus, new means to enhance the effectiveness of this therapy are urgently needed.

Regulation by microRNAs (miRs), small noncoding RNAs that function as posttranscriptional regulators of gene and protein expression, is known to contribute to diverse cellular processes in both normal and cancer cells. While p53 is known to regulate miRs that contribute to its function $(23,24)$, the identity and contribution of p63- and p73-regulated miRs have not been clearly defined. Surprisingly, we find that a substantial fraction of miRs regulated by $\mathrm{p} 63$ in fact target $\mathrm{p} 73$ for inhibition. Detailed analysis reveals one such miR, miR-193a-5p, to be a direct transcriptional target repressed by $\mathrm{p} 63$ and activated by proapoptotic $\mathrm{p} 73$ isoforms. As a result, chemotherapy treatment induces this miR through a $\mathrm{p} 63$ / p73-dependent mechanism in SCC cells, which promotes chemoresistance through feedback control of p73. We exploit this finding as therapeutic proof of principle, demonstrating that inhibition of this miR decreases tumor cell survival and dramatically enhances p73-dependent chemosensitivity both in vitro and in vivo. These findings demonstrate that targeted disruption of this regulatory circuit, which we believe is novel, may represent a useful approach to inducing chemosensitivity in the absence of functional p53.

\section{Results}

A subset of p63-regulated miRstarget p73. Expression of p63 is correlated with the response to chemotherapy in human cancers including SCC and some breast cancers $(25,26)$. To identify miRs regulated by $\mathrm{p} 63$, we ablated endogenous $\mathrm{p} 63$ expression by lentiviral RNA interference, followed by global miR expression profiling using the Exiqon Locked Nucleic Acid (LNA) Platform (Figures 1, A and B, and ref. 27). We performed this initial experiment in JHU-029 SCC cells, a human tumor-derived line that expresses high levels of endogenous $\Delta \mathrm{Np} 63 \alpha$, which is the major p 63 isoform expressed in both normal basal epithelial cells and the majority of human SCCs (7). At 48 hours, the large majority of miRs identified as reproducibly regulated by p 63 were upregulated following p 63 knockdown, consistent with the established ability of $\mathrm{p} 63$ to function as an endogenous transcriptional repressor (Figure 1B; the full list of significantly regulated miRs is provided in Supplemental Table 1; supplemental material available online with this article; doi:10.1172/JCI43897DS1) (8, 28).

Remarkably, examination of predicted target genes of these p63regulated miRs using a combination of in silico resources (TargetScan, MicroCosm, and miRANDA) revealed that 3 of the top 10 most highly regulated miRs, miR-193a-5p, miR-602, and miR-765, were predicted to target the p73 3' UTR (Figure 1B and Supplemental Table 1) (29-31). Of note, only about $3 \%$ of all the miRs represented on this array are predicted to target $p 73$. Following direct validation of p63-dependent regulation of these 3 endogenous miRs (Figure 1C), we then tested their ability to regulate gene expression via the $\mathrm{p} 733^{\prime}$ UTR using a reporter construct containing this UTR fused to luciferase. Cotransfection of this reporter with the corresponding synthetic miR duplexes (miR mimics) in each case inhibited luciferase expression relative to the control reporter lacking the p73 3' UTR (Figure 1D). Consistent with these findings, knockdown of p63, which increases expression of these endogenous miRs, inhibited UTR reporter expression, while overexpression of $\Delta \mathrm{Np} 63 \alpha$ led to a UTR-dependent increase in luciferase expression (Figure 1E and Supplemental Figure 1B). As a specificity control, we then repeated these experiments in cells following lentiviral-mediated knockdown of endogenous Drosha, an RNase III-type endonuclease required for miR nuclear processing. Knockdown of Drosha completely abolished the ability of either p63 overexpression or endogenous p63 ablation to regulate gene expression through the p73 3' UTR (Figure 1E). Thus, we have uncovered a miR-dependent mechanism controlled by $\mathrm{p} 63$ that appears to regulate the key chemosensitivity factor $\mathrm{p} 73$.

Regulation of miR-193a by $p 63$ and $p 73$ mediates its induction following chemotherapy. The miR-193a-5p (hereafter miR-193a) demonstrated the strongest regulation of the p $733^{\prime}$ UTR, and we next studied its physiological regulation in more detail. Consistent with the ability of p63 to repress this $\mathrm{miR}$, its expression correlated inversely with that of $\mathrm{p} 63$ in normal epithelium versus SCC, with the former expressing high levels of the miR and relatively lower p63 levels and the latter showing high p63 levels and low miR levels (Supplemental Figure 1C). This miR is highly conserved in mouse and rat and is designated miR-193* in these species (Supplemental Figure 2A and ref. 32). We showed using lentiviral p63 knockdown that this miR was repressed by endogenous p63 in human and murine squamous carcinoma cells, and in immortal human primary keratinocytes, but not in A549 cells, which do not express endogenous p63 (Figure $2 \mathrm{~A}$ and ref. 8). Conversely, $\Delta \mathrm{Np} 63 \alpha$ overexpression led to repression of miR-193a, and this effect was abolished by introducing a point mutation in p63, R304W, that abrogates the ability of p63 to bind DNA (Figure 2B, Supplemental Figure 2B, and ref. 33). To test regulation of this miR by endogenous $\mathrm{p} 63$ in vivo, we established mice expressing a conditional $p 63$-null allele $\left(p 63^{l o x}\right)(3)$ and a tamoxifeninducible Cre/ER fusion transgene (K14-Cre/ER) (34). Tamoxifen treatment led to a significant decrease in p63 in the epidermis of transgene-expressing mice compared with controls, which was accompanied by robust induction of endogenous miR-193* (Figure 2C). We next examined the relationship between expression of endogenous $\mathrm{p} 63$ and miR-193a in human primary head and neck SCCs (HNSCCs). As predicted, p63 was highly expressed in these tumors, and p63 mRNA levels were strongly inversely correlated with those of miR-193a in these specimens $(P<0.001)$ (Figure 2D). Taken together, these data show that $\mathrm{p} 63$ is an endogenous repressor of this miR in normal human and murine cells and in the context of tumor-specific p63 overexpression.

Both p63 and p73 are thought to contribute to chemosensitivity in cancer cells by regulating a common subset of genes $(8,16,35)$. Therefore, we tested miR-193a regulation by $\mathrm{p} 73$. We first generated cells expressing tetracycline-inducible TAp73 $\beta$, a major p73

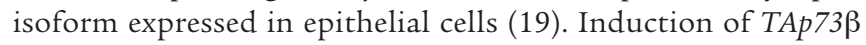
mRNA and protein are detectable within 1 hour following addition of tetracycline in these cells, while induction of miR-193a was detectable within 4 hours, coincident with upregulation of the direct p73 transcriptional target gene PUMA (Figure 2E). In order to test the ability of endogenous $\mathrm{p} 73$ to regulate this miR, we treated both human and murine SCC cells with cisplatin, which is known to activate p73-dependent transcription (refs. 11, 35, 36, and Supplemental Figure 2C). Indeed, in both murine and human cells, cisplatin treatment significantly induced expression of this miR (Figure 2F and Supplemental Figure 2D, respectively). To ascertain whether this induction was mediated via endogenous p73, we ablated p73 expression by lentiviral RNAi prior to cisplatin treatment (Supplemental Figure 2E). Induction of the miR in 


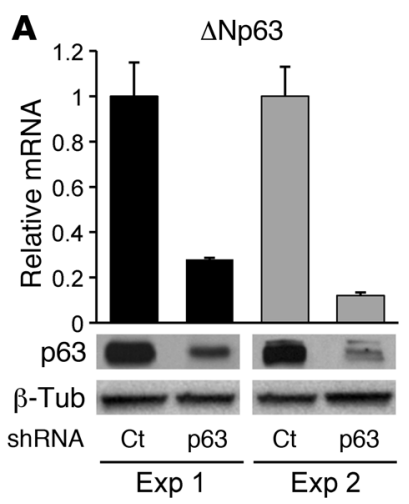

C

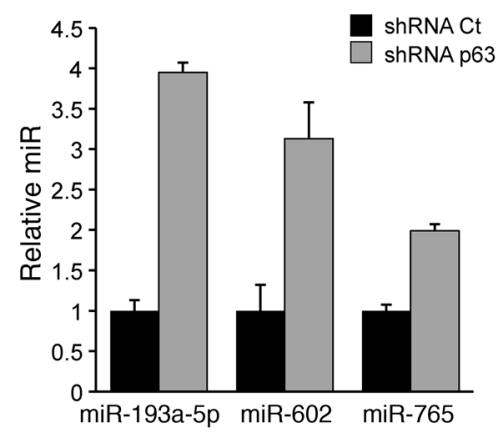

B

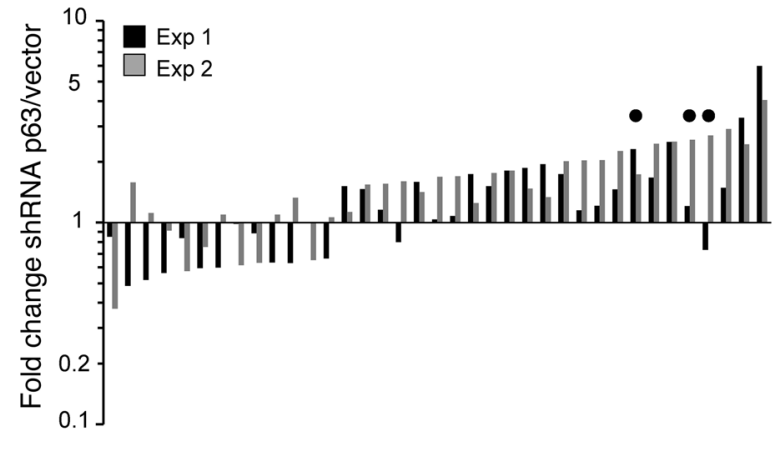

D

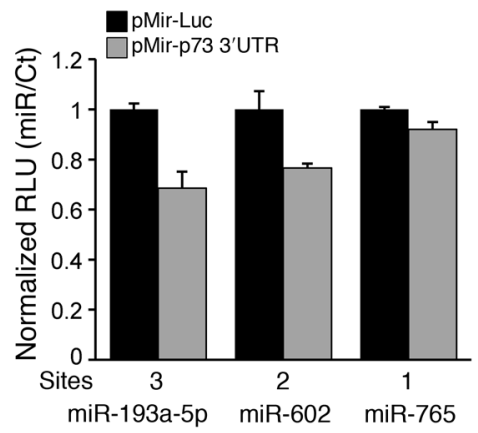

$\mathbf{E}$
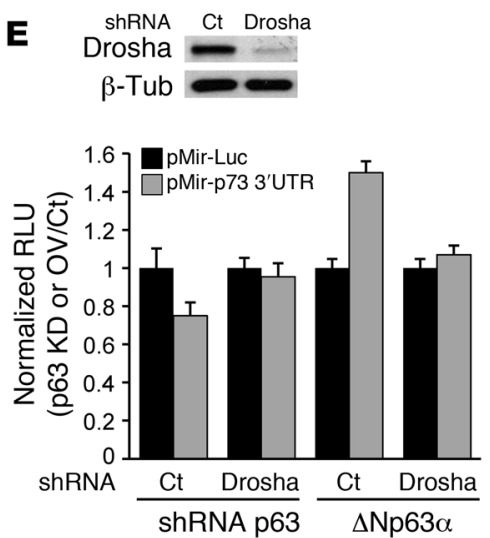

Figure 1

p63-regulated miRs target p73. (A) Knockdown of endogenous p63 RNA (top) and protein (bottom) by p63-directed or control (Ct) lentiviral shRNA in JHU-029 human SCC cells at 48 hours, in duplicate experiments (Exp). $\beta$-Tubulin ( $\beta$-Tub) loading control. (B) Array analysis showing the fold change and direction of change for all miRs regulated at 1.5 -fold or more in p63-ablated versus control samples shown in A. Circles show miRs predicted to target p73. (C) Validation of p63 repression of miRs targeting p73 by real-time quantitative RT-PCR (QRT-PCR) at 72 hours after lentiviral p63-directed or control shRNA expression in JHU-029 cells. (D) The p63-regulated miRs repress gene expression via the p73 3' UTR. Cotransfection of the indicated miR mimics or control (scrambled) miR, together with the UTR-reporter or control reporter; results show relative luciferase units (RLU) normalized to the control miR. Note that repression correlates with the number of predicted seed-binding sequences (Sites) for each miR. (E) A miR-dependent mechanism for regulation of the p73 3' UTR by p63. Lentiviral shRNA knockdown of Drosha followed by cotransfection of the UTR or control reporter, together with either a p63 shRNA or $\triangle N p 63 \alpha$ cDNA or their respective controls in JHU-029 cells. RLU values expressed as p63 knockdown/control or $\Delta$ Np63 $\alpha$ overexpression/control (OV/Ct). Above, immunoblot shows efficient Drosha knockdown. All error bars show SEM for triplicate measurements from representative experiments.

response to cisplatin was abolished in both murine and human cells by p73 knockdown, thus confirming endogenous p73-mediated upregulation of this miR (Figure 2F and Supplemental Figure 2D). Given that p73 activation following cisplatin treatment is thought to rely on both p63 degradation (Supplemental Figure $2 \mathrm{E}$ ) and $\mathrm{p} 73$ posttranslational modification $(11,21,22)$, these findings support a $\mathrm{p} 63 / \mathrm{p} 73$-dependent mechanism for miR-193a induction following cisplatin treatment in SCC.

In contrast to these findings, we observed that this miR was not regulated by endogenous p53. Following treatment of WT and $p 53^{-/-}$mouse embryo fibroblasts (MEFs) with doxorubicin to induce DNA damage, we observed induction of direct p53 target genes including Bax and Noxa in a p53-dependent manner (Figure $2 \mathrm{G}$ ). However, no induction of miR-193* was observed in the presence of p53. Similarly, no induction of miR-193a was seen in human primary keratinocytes following doxorubicin treatment despite substantial induction of direct p53 transcriptional target genes (Supplemental Figure 2F). Of note, in the $p 53^{-/-}$MEFs, this miR was induced together with p73 following DNA damage (Fig- ure $2 \mathrm{G}$ ). Taken together, these data show that miR-193a is induced in response to chemotherapy in a p53-independent but p63/p73dependent manner. These findings led us to hypothesize that miR193a induction by chemotherapy may function to induce chemoresistance through feedback inhibition of $\mathrm{p} 73$.

Direct transcriptional regulation of miR-193a by $p 63$ and $p 73$. The miR-193a localizes to an intergenic region on human chromosome 17q. Alignment of the syntenic genomic loci for human, mouse, and rat using the Vista software (37) demonstrates that 2 regions of highest conservation within this locus correspond to the miR itself and a second region 10-kb upstream of miR-193a (Figure $3 \mathrm{~A})$. Both of these conserved regions contain CPG islands, suggesting they may contain essential transcriptional regulatory elements. ChIP for endogenous p63, however, revealed robust binding only within the highly conserved region $10-\mathrm{kb} 5^{\prime}$ of miR-193a in JHU029 cells (Figure $3 \mathrm{~A}$ ). This same region, but not adjacent ones, was also bound by endogenous $\mathrm{p} 63$ in human primary keratinocytes (Figure 3B). As an additional specificity control, we overexpressed epitope-tagged WT $\Delta \mathrm{Np} 63 \alpha$ or the DNA-binding defective point 
A

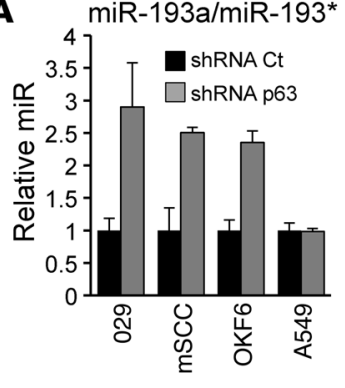

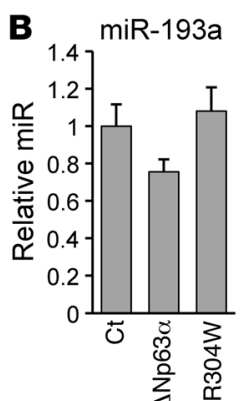

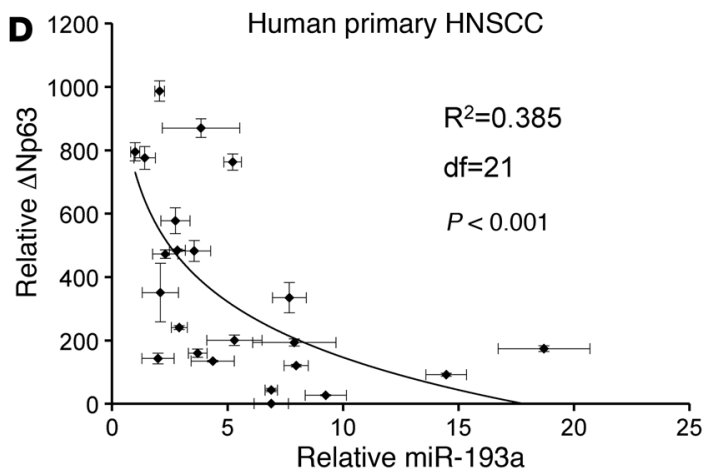

$\mathbf{F}$

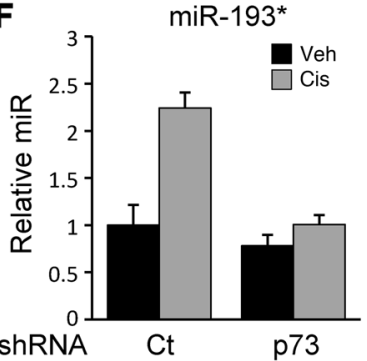

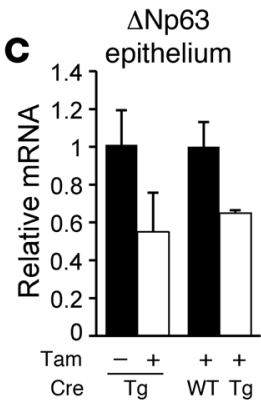

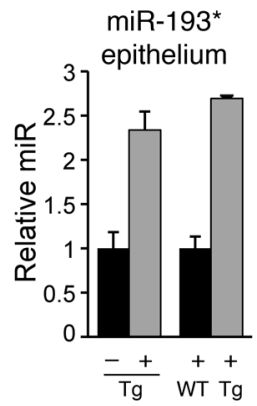

$\mathbf{E}$
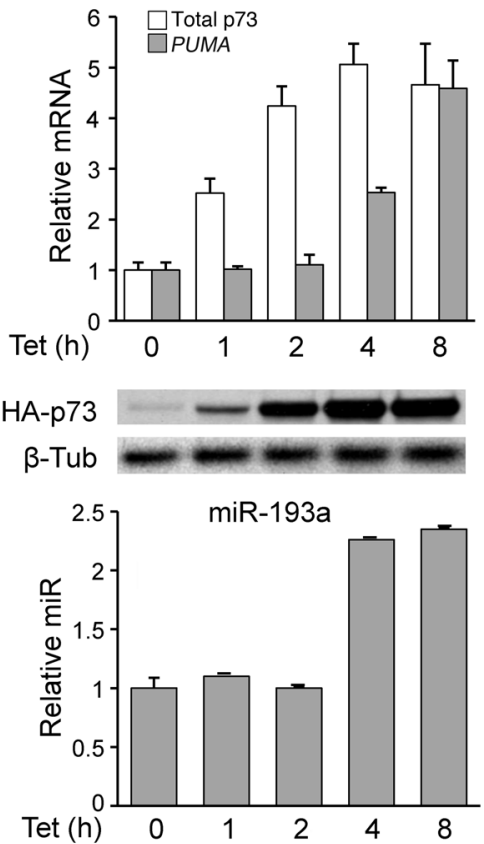
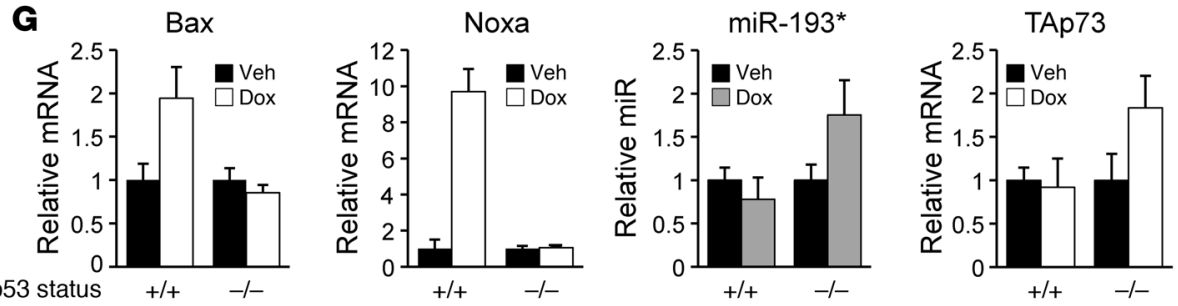

Figure 2

Endogenous p63 and p73 mediate miR-193a regulation by chemotherapy. (A) Regulation of human miR-193a-5p (hereafter miR-193a) and murine miR-193* by endogenous p63, measured by QRT-PCR 48 hours after lentiviral p63 knockdown in JHU-029 cells (029), murine SCC cells (mSCC), human immortal primary keratinocytes (OKF6), and control A549 cells, which do not express p63. (B) Repression of endogenous miR193a by p63 requires DNA binding. Retroviral expression of WT or non-DNA-binding $\triangle$ Np63 $\alpha$ mutant (R304W) was followed by QRT-PCR. (C) Regulation of murine miR-193* by endogenous $p 63$ in vivo. Homozygous $p 63^{\text {flox }}$ mice with or without expression of the K14-Cre/ER transgene (Tg) were treated with tamoxifen (Tam) or vehicle control for 5 days, and epidermal cells were collected 21 days later for RNA analysis by QRTPCR. (D) Inverse correlation between p63 expression and miR-193a expression in human primary HNSCC specimens, assessed by QRT-PCR. df, degrees of freedom. (E) Rapid upregulation of endogenous PUMA (top) and miR-193a (bottom) following tetracycline-induced (Tet) TAp73 expression in JHU-029 cells. Middle panels show induced TAp73 expression by immunoblot. (F) Endogenous p73-dependent induction of miR$193^{*}$ in murine SCC cells by cisplatin. Following preinfection with lentiviral TAp73 shRNA or control, cells were treated with cisplatin (Cis, $4 \mu \mathrm{M}$, 24 hours) or vehicle control (Veh) prior to RNA analysis. (G) Regulation of miR-193* is independent of p53. WT or p53-/- E1A-immortalized MEFs were treated with doxorubicin (Dox) $(0.2 \mu \mathrm{M}, 12$ hours) or vehicle control prior to RNA analysis. Bax and Noxa are induced in a p53-dependent manner, whereas miR-193* induction correlates only with TAp73. All error bars show SEM for triplicate measurements. 

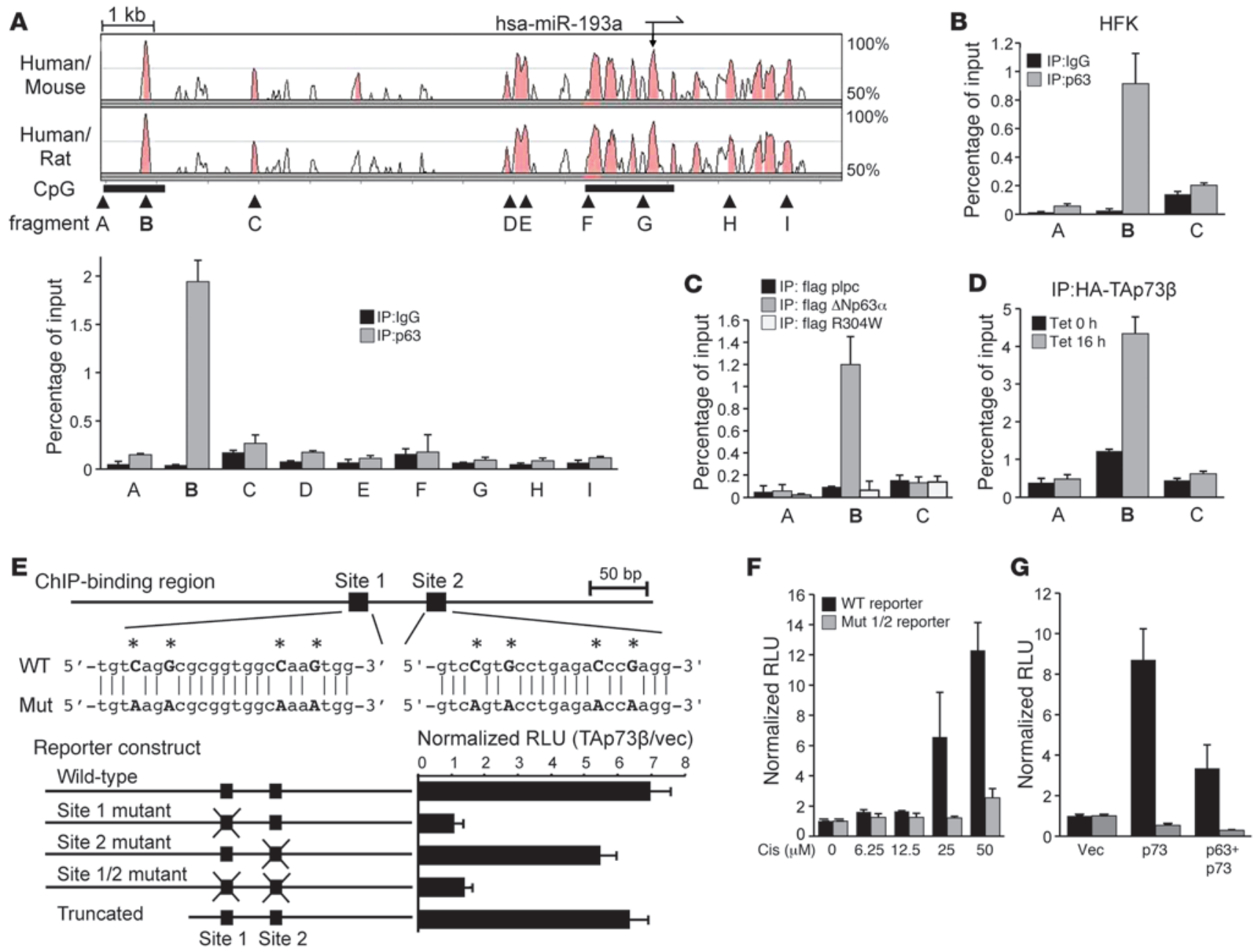

WT 5'-tgtCagGcgcggtggcCaagtgg-3' 5'-gtcCgtGcctgagaCccGagg-3'

Mut 5'-tgtaagAcgCggtggCAaatgg-3' 5'-gtcAgtAcctgagaAcCAagg-3 '

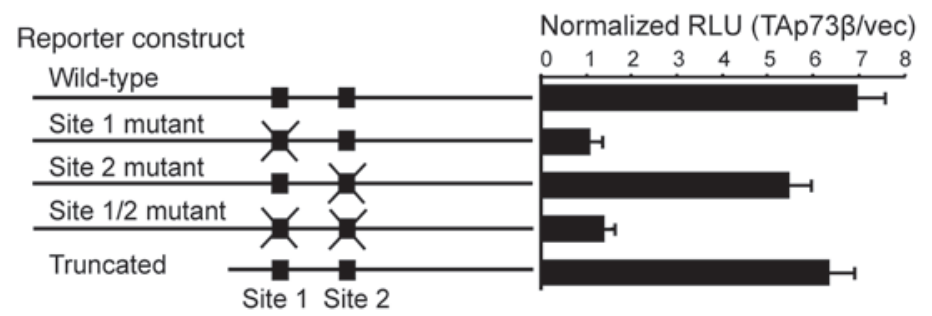

Figure 3

miR-193a is a direct transcriptional target of p63 and p73. (A) Direct binding of p63 within a conserved CpG island upstream of miR-193a. Top: cross-species Vista alignment; peaks show degree of homology; arrows at top show position and orientation of miR-193a; black bars below indicate CpG islands; arrowheads show location of ChIP fragments. Bottom: ChIP results in JHU-029 cells for the indicated fragments. (B) ChIP in primary human foreskin keratinocytes (HFK) for the fragments indicated in A. (C) ChIP showing specific binding of WT $\Delta$ Np63 $\alpha$ but not the DNA-binding-defective mutant to the conserved region B in JHU-029 cells. (D) ChIP showing binding of tetracycline-induced p73 to region B. Binding in the absence of tetracycline results from leaky p73 expression. (E) Transcriptional activation of a miR-193a regulatory region requires a conserved p63/p73 DNA-binding motif (site 1). Top: schematic of ChIP-binding region B with putative binding motifs, their sequences, and introduced point mutant (Mut) sequence indicated. Bottom: cotransfection of TAp73 $\beta$ with a luciferase reporter containing the indicated fragments. (F) Dose-dependent induction of the miR-193a reporter by cisplatin (Cis) (24 hours) requires the p63/p73 DNA-binding motif. JHU-029 cells were treated as indicated 12 hours after reporter transfection. (G) p63 represses p73-dependent activation of the miR-193a reporter. Cotransfection of the indicated reporter with vector, TAp73 $\beta$ (p73) and vector, or TAp73 $\beta$ and $\Delta$ Np63 $\alpha$ (p63) in JHU-029 cells. All error bars show SEM for triplicate measurements.

mutant $\Delta \mathrm{Np} 63 \alpha, \mathrm{R} 304 \mathrm{~W}$, showing by ChIP that p63 was bound via its DNA-binding domain to this site (Figure 3C). Consistent with our model for miR-193a regulation by $\mathrm{p} 63 / \mathrm{p} 73$, we also observed $\mathrm{p} 73$ binding exclusively at this region following tetracycline-regulated p73 expression (Figure 3D), as well as endogenous p73 binding following cisplatin treatment (Supplemental Figure $3 \mathrm{~A})$. Thus, $\mathrm{p} 63$ and $\mathrm{p} 73$ bind specifically to a region within the miR-193a genomic locus.

Both $\mathrm{p} 63$ and $\mathrm{p} 73$ are thought to bind DNA via a shared consensus nucleotide sequence motif (15). We identified 2 such motifs within the ChIP-identified p63/p73-binding region (Figure $3 \mathrm{E}$ ). The 500-bp genomic fragment containing these motifs was suf- ficient to confer strong transcriptional induction of a luciferase reporter in response to $\mathrm{p} 73$ expression (Figure 3E). By introducing point mutations of key nucleotides required for transcription factor binding within these motifs, we showed that only the distal site was required for p73-mediated transcriptional regulation (Figure $3 \mathrm{E})$, consistent with the fact that only this site was evolutionarily conserved (Supplemental Figure 3B). Furthermore, a 5' truncated reporter that still contained the binding motifs was induced at levels comparable to the full-length reporter (Figure 3E). To test endogenous p73-dependent induction of this regulatory sequence, we treated JHU-029 cells harboring the reporter with cisplatin, which as shown above (Supplemental Figure 2C) activates p73- 

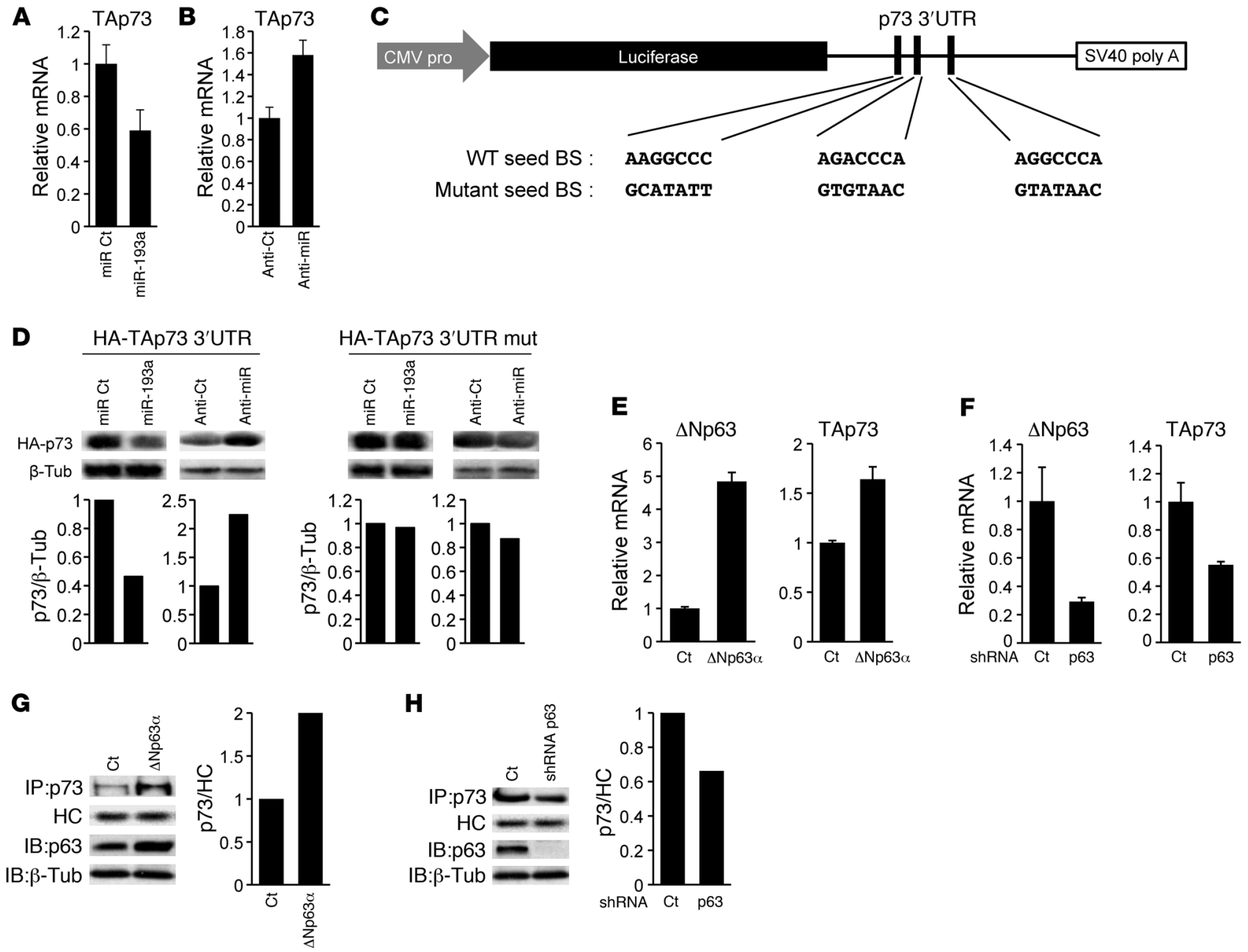

H

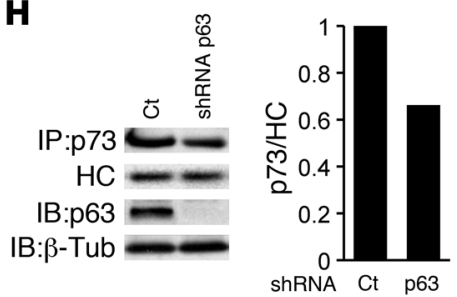

\section{Figure 4}

Direct inhibition of p73 by miR-193a is opposed by p63. (A) Endogenous p73 mRNA is inhibited 48 hours following transfection of JHU-029 cells with a miR-193a mimic compared with a scrambled control miR. (B) Endogenous $p 73$ mRNA is derepressed 48 hours following transfection of JHU-029 cells with a miR-193a antagomir (anti-miR) compared with scrambled control (anti-Ct) antagomir. (C) Schematic of the p73 3' UTR reporter construct showing putative miR-193a seed-binding sequences (WT seed BS) and nucleotide changes introduced in the mutant reporter (mutant seed BS). (D) UTR-dependent regulation of p73 protein requires miR-193a seed-binding sequences. Immunoblots of lysates following cotransfection of a TAp73 $\beta$ cDNA linked to the WT (left 2 panels) or mutant (mut, right panels) 3' UTR, together with either a miR-193a mimic, a specific antagomir (anti-miR), or respective controls. Bar graphs below show densitometry. Blots are representative of triplicate experiments. (E) Endogenous p73 mRNA is regulated by p63. Retroviral $\triangle N p 63 \alpha$ or vector control were expressed in JHU-029 cells followed by QRT-PCR for $\Delta$ Np63 or TAp73. (F) Endogenous p63-mediated regulation of $p 73$ mRNA. Infection of JHU-029 cells with lentiviral p63 shRNA or scrambled control, followed by QRT-PCR for $\Delta$ Np63 or TAp73. (G) Regulation of p73 protein by p63. Retroviral $\Delta$ Np63 $\alpha$ expression as in E was followed by IP/immunoblot (p73) or immunoblot (p63). Right, densitometry analysis for p73. Heavy chain (HC) loading control. (H) Endogenous p63-mediated regulation of p73 protein. Lentiviral p63 knockdown as in F, followed by analysis as in $\mathbf{G}$. Error bars show SEM for triplicate measurements.

dependent transcription in these cells. Cisplatin treatment led to significant activation of the WT reporter, while it had little or no effect on the reporter bearing mutations of the consensus p63/p73-binding motifs (Figure 3F). Finally we showed that p63 expression was sufficient to repress $\mathrm{p} 73$-dependent induction of this reporter (Figure 3G) (8). Together, these experiments suggest that $\mathrm{p} 63$ and $\mathrm{p} 73$ bind directly to a consensus motif upstream of the mature miR-193a sequence, thereby directly regulating miR193a transcription in response to chemotherapy.

miR-193 a mediates feedback inhibition of 773 , which is opposed by $p 63$. We next wished to verify whether miR-193a was a direct inhibitor of endogenous $\mathrm{p} 73$, suggesting it may be a determinant of chemoresistance, and whether p63 would oppose this effect through miR-193a repression, consistent with the association between p63 expression and chemosensitivity $(25,26)$. As predicted by this model, transfection of a miR-193a mimic suppressed endogenous p73 mRNA levels (Figure 4A), while expression of a specific antagomir caused an increase in endogenous $p 73$ mRNA in both human and murine cells (Figure 4B and Supplemental Figure 4A, respectively). Three predicted seed-binding sequences for miR-193a are present within the p73 3' UTR (Figure 4C and Supplemental Figure 4B). To address direct $\mathrm{p} 73$ regulation by miR-193a, we 

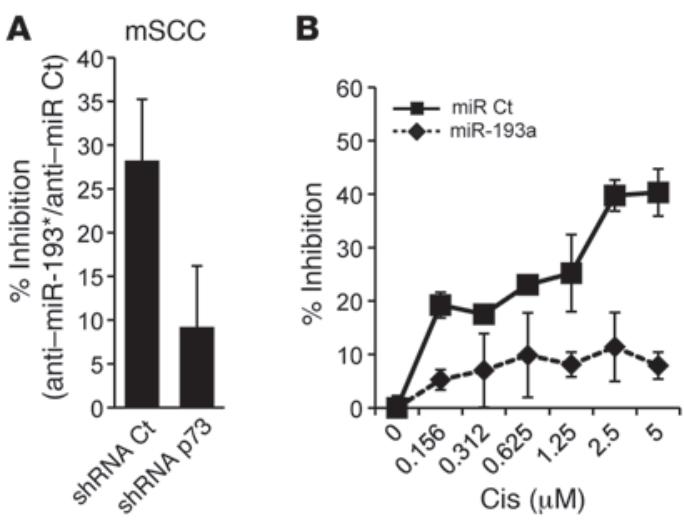
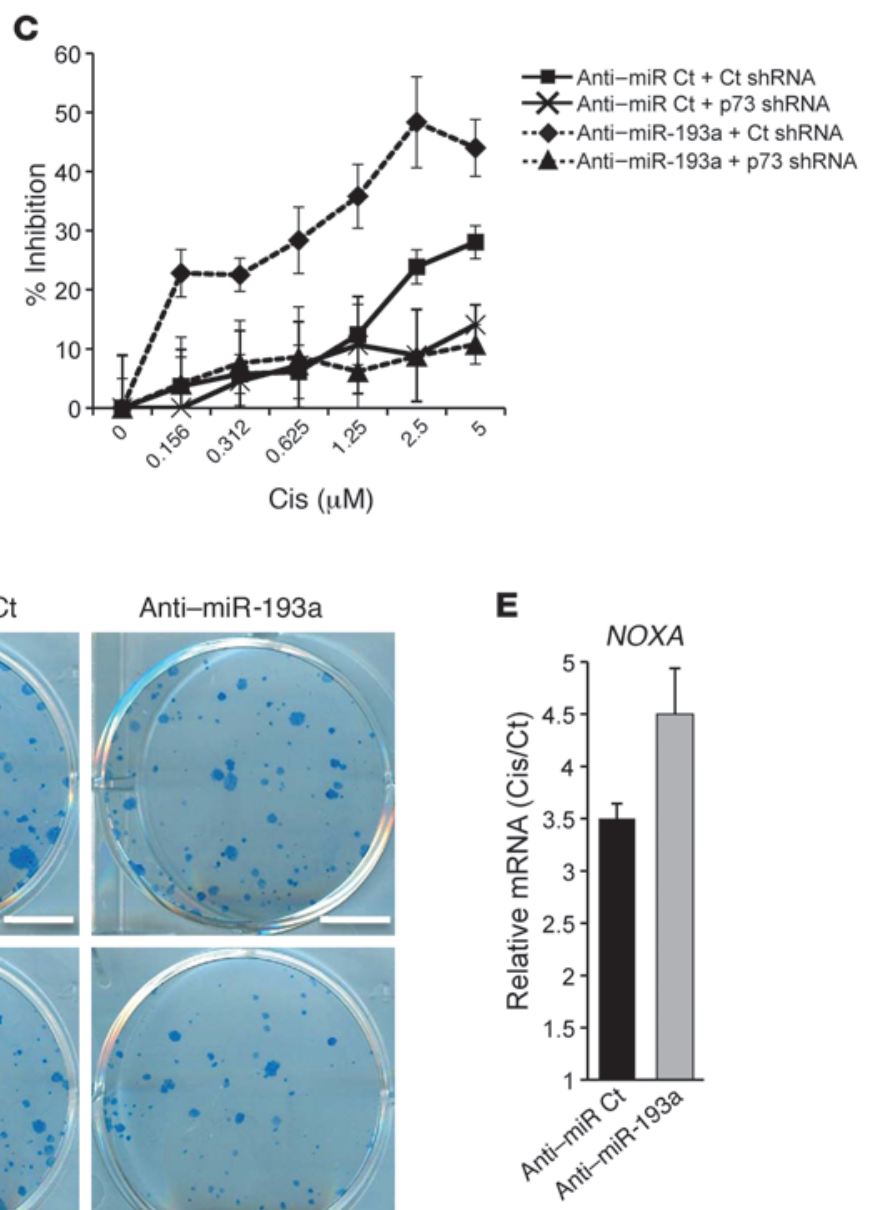

$\mathbf{E}$
D

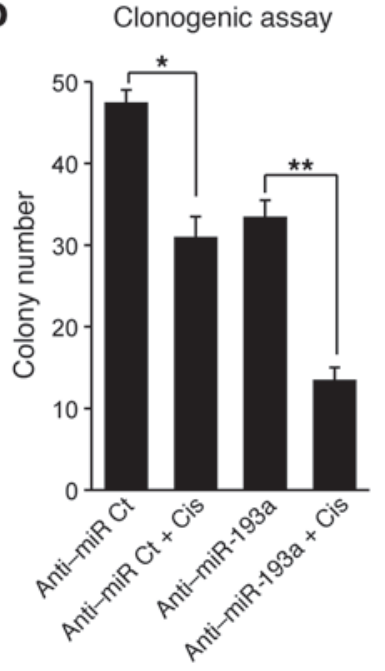

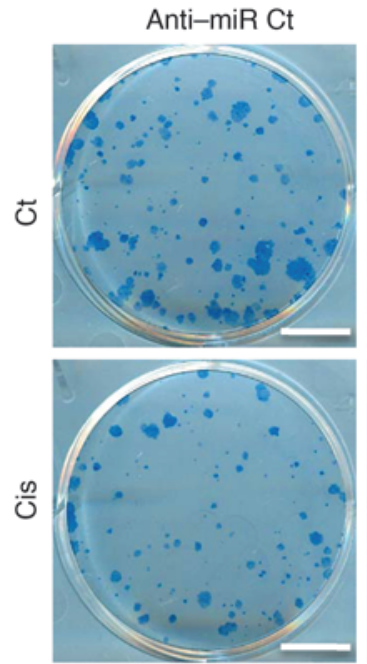

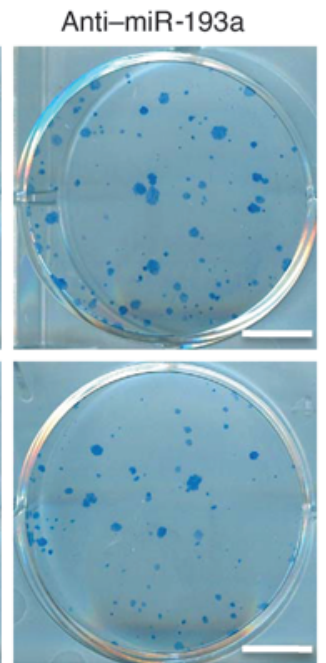

\section{Figure 5}

miR-193a regulates cell viability and chemosensitivity through p73. (A) p73-dependent inhibition of viable cells following miR-193* antagomir (anti$\mathrm{miR}$ ) treatment. Murine SCC (mSCC) cells were infected with lentiviral p73 shRNA or control, then treated with a miR-193* antagomir or Scrambled control followed by cell counts at 72 hours. (B) miR-193a abrogates cisplatin-induced growth inhibition. JHU-029 cells were pretreated with miR193a mimic or scrambled control (miR Ct) for 24 hours followed by 1 hour cisplatin treatment, and cell counts were determined by XTT assay 72 hours later. (C) Enhancement of cisplatin sensitivity by the miR-193a antagomir is p73-dependent. JHU-029 cells were infected with lentiviral TAp73 shRNA or control, then treated for 24 hours with a miR-193a antagomir or scrambled control (anti-miR Ct) prior to 1 hour cisplatin treatment and cell counts 72 hours later. (D) Endogenous miR-193a controls cisplatin-mediated clonogenic suppression. JHU-029 cells were treated for 24 hours with a miR-193a antagomir (anti-miR-193a) or scrambled control (anti-miR Ct) prior to cisplatin treatment ( $0.5 \mu \mathrm{m}, 1 \mathrm{hour}$ ), then plated at clonal density for colony counts. Scale bar: $1 \mathrm{~cm}$. ${ }^{*} 34.7 \%$ repression; ${ }^{* *} 64.5 \%$ repression. (E) Control of p73 activity by endogenous miR-193a. Cells treated as in D were harvested at 24 hours after cisplatin for analysis of the p73 target gene NOXA by QRT-PCR. Error bars show SEM.

introduced point mutations within these sequences. As predicted, the p73 3' UTR reporter inhibition observed following cotransfection with a miR-193a mimic was completely abolished when a reporter containing the mutant UTR was used instead (Supplemental Figure 4C). To test regulation by endogenous miR-193a, we cotransfected a miR-193a antagomir into SCC cells with either the WT or mutant UTR reporter. Consistent with our model, antagomir-mediated upregulation of the UTR reporter was abolished by mutations within the miR-193a seed-binding sequences (Supplemental Figure 4D).

These same effects of miR-193a were observed on the $\mathrm{p} 73$ protein itself. To verify direct effects of miR-193a on $\mathrm{p} 73$ protein expression, we expressed a p73 cDNA that included either the WT or mutant 3' UTR. Expression of p73 protein was consistently inhibited when it was coexpressed with the miR-193a mimic, and this effect was abolished by mutation of the seed-binding sequences for this miR within the p73 3' UTR (Figure 4D). Most importantly, treatment with a miR-193a antagomir demonstrated that endogenous miR-193a inhibited p73 protein expression through the miR-193a seed-binding sequence in SCC cells (Figure 4D). Together these experiments demonstrate that miR-193a is a direct, endogenous regulator of $\mathrm{p} 73$. Most notably, the antagomir experiments demonstrate that miR-193a is a constitutive inhibitor of p73 in SCC cells.

Overexpression of $\mathrm{p} 63$, as is observed in SCC, is predicted to contribute to increased p73 through miR-193a suppression. Indeed, overexpression of $\Delta \mathrm{Np} 63 \alpha$, the major $\mathrm{p} 63$ isoform expressed in human SCC, led to induction of endogenous $p 73 \mathrm{mRNA}$ (Figure 4E). 

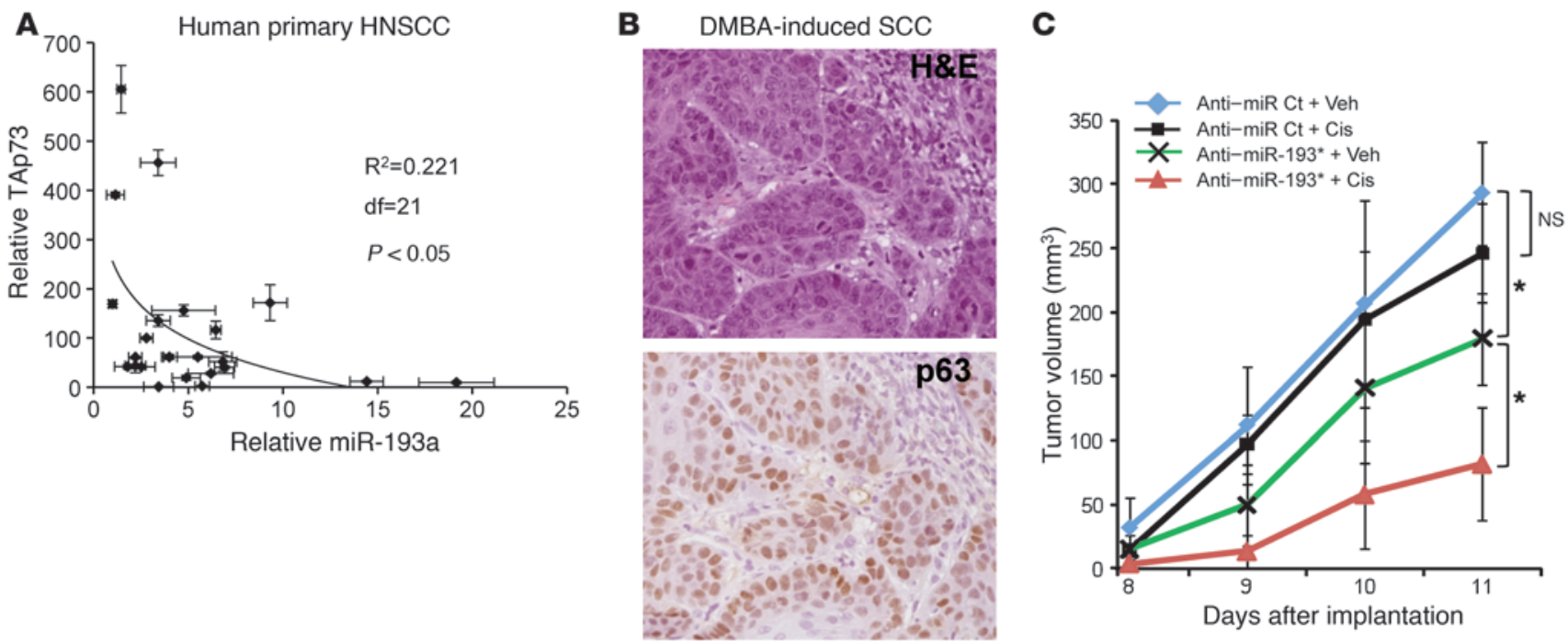

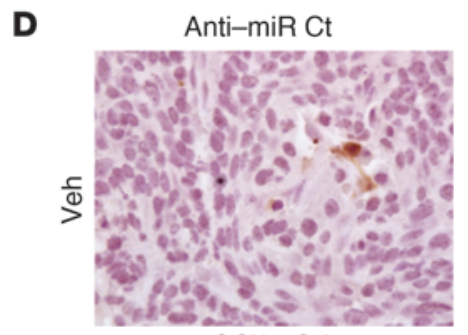

$6.6 \% \pm 3.4$

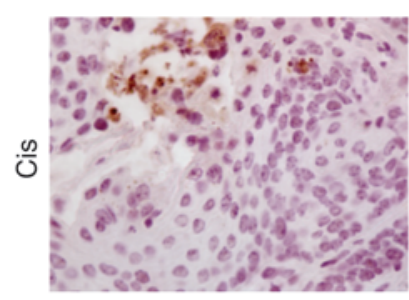

$13.2 \% \pm 4.8$

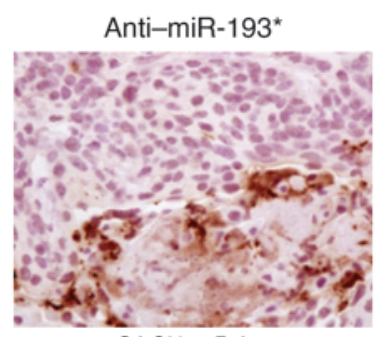

$24.6 \% \pm 5.4$

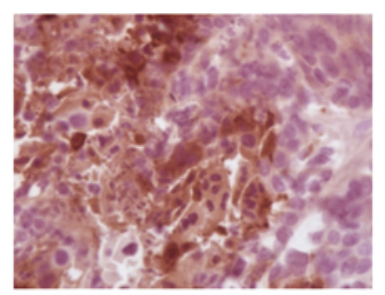

$51.2 \% \pm 8.8$
E

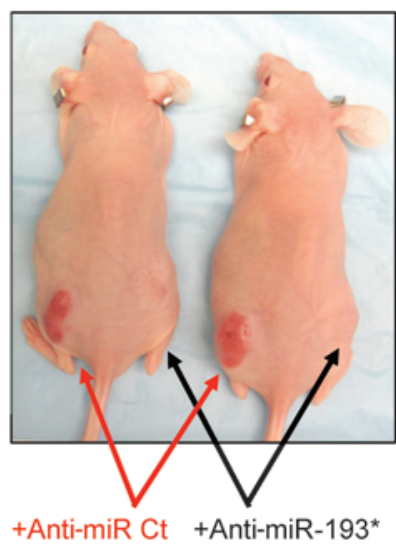

Figure 6

In vivo control of tumor progression and chemosensitivity by miR-193a. (A) Inverse correlation between miR-193a and p73 expression in primary human HNSCC specimens, assessed by QRT-PCR. (B) Murine SCCs resemble human SCC, assessed by histology (H\&E) and p63 immunohistochemical staining. Original magnification, $\times 200$. (C) Anti-miR-193* inhibits tumor growth in the absence of cisplatin, and it enhances cisplatin sensitivity. Primary disaggregated SCC was treated with miR-193* antagomir or scrambled control, then implanted into nude mice, followed by cisplatin treatment $(5 \mathrm{mg} / \mathrm{kg})$ or vehicle. $n=10$ per arm. ${ }^{*} P<0.05$ by 2 -way repeated measures ANOVA. (D) Increased expression of activated caspase-3 (brown staining) in vivo following antagomir-mediated miR-193* inhibition compared with control antagomir treatment, both in the presence and absence of cisplatin treatment. Original magnification, $\times 200$. The percentage of cleaved caspase-3-positive cells \pm SEM is shown below each panel, based on 100 cells counted in 3 representative fields. (E) Representative mice following cisplatin treatment.

This finding correlated with ability of $\Delta \mathrm{Np} 63 \alpha$ to repress endogenous miR-193a (Figure 2B). Similarly, lentiviral knockdown of endogenous p63, which induces miR-193a expression (Figure 2), suppressed endogenous $p 73$ mRNA expression (Figure 4F). Furthermore, in each experiment the effects we observed on endogenous $p 73$ mRNA paralleled the effects on the UTR reporter. Thus, overexpression of $\Delta \mathrm{Np} 63 \alpha$ induced the WT p73 3' UTR reporter, but had no effect on the mutant reporter (Supplemental Figure $5 \mathrm{~A})$, while p63 knockdown suppressed only the WT reporter (Supplemental Figure 5B). As predicted, p73 protein expression increased following $\Delta \mathrm{Np} 63 \alpha$ overexpression (Figure $4 \mathrm{G}$ ) but decreased following knockdown of endogenous p63 (Figure 4H).
Finally, in keeping with the prediction that this miR-dependent mechanism promotes $\mathrm{p} 73$ upregulation in response to tumor-specific p63 overexpression, we observed a significant positive linear correlation between $\mathrm{p} 63$ and p73 levels in primary HNSCC tumors (Supplemental Figure 5C), in agreement with published data (18). These findings position miR-193a as a pivotal regulator of $\mathrm{p} 73$ through p63, suggesting the possibility of a unique contribution to $\mathrm{p} 63 / \mathrm{p} 73$-dependent chemosensitivity.

miR-193 a controls p73-dependent cell viability and chemosensitivity in SCC cells. In direct support of a role for endogenous miR-193a and its murine ortholog in promoting tumor cell viability, treatment of SCC cells with a miR-193* antagomir substantially suppressed 

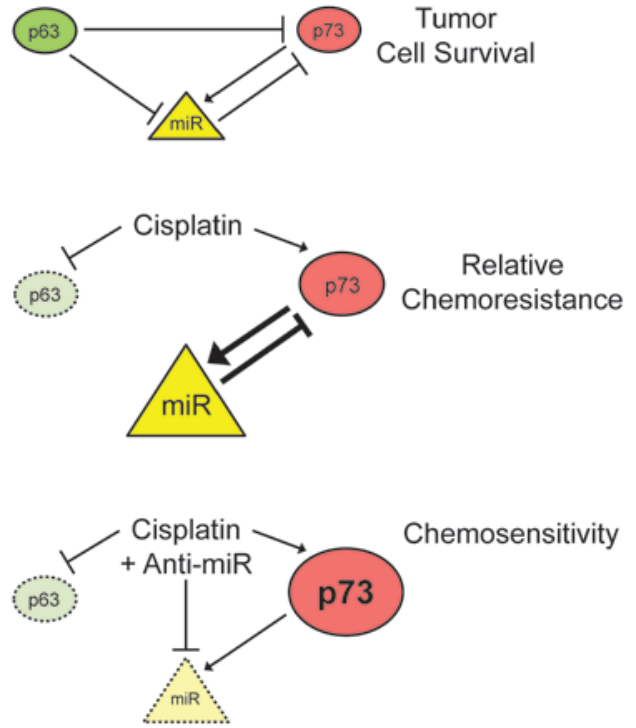

\section{Figure 7}

Model for miR-dependent mechanism mediating p63/p73 crosstalk, tumor cell survival, and chemosensitivity. Top: in SCC, p63 is an inhibitor of $\mathrm{p} 73$ and a transcriptional repressor of miRs that target p73. The miR-193a/193* is a direct transcriptional target of both $\mathrm{p} 63$ and p73 and a direct feedback inhibitor of p73. Middle: cisplatin treatment induces p63 degradation and p73 activation, thereby inducing this miR, which then limits p73-dependent chemosensitivity through direct feedback inhibition. Bottom: this feedback loop is disrupted by miR inhibition, which increases p73 levels and enhances chemosensitivity.

viable cell numbers (Figure 5A) at the same time as it increased p73 levels (Supplemental Figure 4A). Furthermore, the effect of miR inhibition on cell viability was p73 dependent, as it was abolished by lentiviral p73 knockdown (Figure 5A). We next tested the contribution of the $\mathrm{miR}$ in the response to cisplatin, an established activator of p73-dependent transcription and cell death $(11,35$, 36). Indeed, as shown above, treatment of SCC cells with cisplatin led to miR-193a reporter activation (Figure 3) and endogenous miR-193a induction via p73 (Figure 2). We first performed a dose-response experiment in human SCC cells following cisplatin treatment, in the presence of the miR-193a mimic or control. As predicted, chemosensitivity was dramatically decreased in cells in which the miR-193a mimic was expressed, with the greatest differences seen at the highest cisplatin doses (Figure 5B). Furthermore, miR-193a antagomir expression increased chemosensitivity in response to cisplatin (Figure 5C), an effect that was mediated at least in part through a substantial increase in cell death (Supplemental Figure 6A and Supplemental Video 1). Inhibiting endogenous miR-193a also increased chemosensitivity assessed in a clonogenic assay, as cisplatin treatment suppressed colony formation substantially more following antagomir treatment compared with control (Figure 5D). This effect was not limited to JHU-029 cells, as antagomir treatment significantly enhanced cisplatin chemosensitivity in 4 additional human tumor-derived SCC cell lines by clonogenic assay (Supplemental Figure 6B). In keeping with what we had observed in the absence of cisplatin, we found that the effect of the miR-193a antagomir was specifically attributable to regulation of $\mathrm{p} 73$, since little or no difference in chemosensitivity was observed in control versus antagomir-treated cells when lentiviral p73 knockdown was performed prior to cisplatin treatment (Figure 5C). To lend additional support to miR-193a-dependent regulation of $\mathrm{p} 73$ function, we examined transcription of the p73regulated proapoptotic gene NOXA following cisplatin treatment in the presence or absence of the antagomir. This gene was more highly induced in response to cisplatin following inhibition of endogenous miR-193a (Figure 5E). Thus, endogenous miR-193a is a key determinant of tumor cell viability, which is induced by p73 in response to cisplatin, thereby potentiating chemoresistance through its feedback inhibition of p73 itself.

Control of tumor progression and chemosensitivity by miR-193a in vivo. Finally, we sought to assess the contribution of miR-193a to tumor cell survival and chemosensitivity in vivo. We first examined primary human tumors for evidence that this miR contributes to constitutive regulation of $\mathrm{p} 73$. Consistent with this hypothesis, we observed a significant inverse correlation between miR-193a levels and p73 levels in human primary HNSCCs (Figure 6A). To directly test the contribution of this $\mathrm{miR}$ in vivo, we developed a mouse model for SCC. This model, which involves treatment of mice with the tumor promoter 9,10-dimethyl-1,2-benzanthracene (DMBA) (Supplemental Figure 7A), recapitulates the features of human SCC, including squamous differentiation, high-level p63 expression (Figure 6B and Supplemental Figure 7B), and metastasis to local lymph nodes (38). Of note, loss of functional p53 was shown to be a rate-limiting event for tumor formation in this model (38). As shown above, cells derived from these tumors exhibit the same regulation of miR-193* by both endogenous $\mathrm{p} 63$ and p73 as do human cells (Figure 2). Additionally, as in human cells, we found that inhibition of endogenous murine miR-193* potentiated both $\mathrm{p} 73$-dependent chemosensitivity (Supplemental Figure 7, C and D) and the activation of a p73 transcriptional target gene (Supplemental Figure 7E).

We therefore used this model to test the contribution of endogenous miR-193a to both tumor growth and cisplatin sensitivity in vivo. In order to perform rigorously controlled experiments, we used in vivo passaging of primary tumors (Supplemental Figure $7 F)$ so that we could compare effects of cisplatin treatment on the same primary tumor in the presence or absence of the miR-193* antagomir. Thus, disaggregated tumors were reimplanted into multiple mice in the presence of the antagomir or control, followed by treatment with cisplatin or control.

Consistent with a specific effect in potentiating p73 activity in the absence of exogenous DNA damage, miR-193* inhibition alone decreased tumor growth (Figure 6C) in association with increased staining of activated caspase-3, a hallmark of apoptosis (Figure 6D). Most significantly, miR inhibition also strongly enhanced the therapeutic effect of cisplatin. Thus, a cisplatin dose that had little or no effect on control antagomir-treated tumors dramatically attenuated growth of miR-193* antagomir-treated tumors (Figure 6, C and E). Apoptosis contributed to this effect, as a substantial increase in activated caspase- 3 staining was observed in cisplatin-treated tumors exposed to the antagomir compared with control antagomir-treated tumors (Figure 6D). Taken together, these experiments demonstrate a direct regulatory circuit that mediates p63/p73 functional crosstalk through transcriptional control of miR-193a (Figure 7). Expression of this miR controls tumor cell viability in SCC, while miR-193a induction following cisplatin treatment limits the therapeutic effect of this chemotherapy through feedback inhibition of $\mathrm{p} 73$. 


\section{Discussion}

The p53 transcription factor family members are highly homologous and play critical roles in development, DNA damage responses, and tumorigenesis. Consequently, potential mechanisms of functional collaboration and antagonism among family members are much discussed, but with few exceptions are poorly understood. Through our characterization of p63-regulated miRs, we have uncovered what we believe is a new mechanism of $\mathrm{p} 63 / \mathrm{p} 73$ functional crosstalk that mediates induced chemoresistance in SCC. This mechanism involves direct transcriptional regulation of miR193 a by both p63 and p73 as well as miR-mediated feedback regulation to p73 itself. Our finding that p53 does not participate in direct transcriptional regulation of this miR (Figure 2) is in keeping with the somewhat weaker homology observed within its DNA-binding domain compared with p 63 and p $73(14,15)$. Importantly, our data together with other published work suggest that this regulatory circuit may have a particularly prominent role in tumors such as SCC that express both $\mathrm{p} 63$ and $\mathrm{p} 73$ but exhibit mutational inactivation of p53 $(18,19)$. Identifying a p53-independent mechanism linked specifically to chemosensitivity is particularly relevant in SCC, as inactivation of p53 in this tumor subtype is associated with both chemoresistance and a poorer overall prognosis $(39,40)$.

Our study demonstrates that p63 functions as a transcriptional repressor of miR-193a, while p73 functions as a direct transcriptional activator of this miR (Figures 2 and 3 ). These findings are in keeping with the established ability of $\Delta \mathrm{Np} 63 \alpha$, the major isoform expressed in the epithelium, to function as a transcriptional repressor of genes, including $C D K N 1 A$, which are regulated by other p53 family members (28). The ability of TAp73 to regulate this miR in response to cisplatin is also in agreement with a large body of data demonstrating a critical role for p73-dependent transactivation in the DNA damage response and chemosensitivity (reviewed in ref. 41). It is of note that the feedback loop from miR-193a to p73 potentially involves UTR-dependent regulation of multiple p73 isoforms, including $\Delta \mathrm{Np} 73$ isoforms. Nevertheless, our data suggest that feedback to TAp73 is most relevant in SCC, as shRNA directed specifically against TAp73 isoforms reverses effects of miR193 a inhibition in both human and murine SCC cells (Figure 5).

Transcriptional repression of miR-193a by $\mathrm{p} 63$ may begin to explain the somewhat paradoxical association of elevated p63 expression in tumor cells with chemosensitivity $(25,26)$. Expression of p63 is linked to proliferation, cellular survival, and regenerative potential, which likely explains selection for its overexpression and genomic amplification in SCC. Our findings suggest that p63 expression contributes to chemosensitivity through direct repression of miR-193a and consequent upregulation of $\mathrm{p} 73$. This regulatory circuit may also explain the unfortunately limited success of chemotherapy in SCC. Thus, treatment with cisplatin leads to p63 degradation and p73 activation $(11,21)$, which together induce miR-193a and inhibit p73, thereby limiting the therapeutic response. We show directly that this induced chemoresistance mechanism can be overcome by inhibiting miR expression in vivo, which converts a completely ineffective chemotherapy dose to a potent suppressor of tumor progression (Figure 6).

Given that our findings point to miR-193a as a key regulator of p63/p73-dependent chemosensitivity, it is reasonable to ask whether expression of this miR itself might be useful in predicting the response of SCCs to chemotherapy. Indeed, we found that primary HNSCC tumors exhibiting high levels of miR-193a are likely to have lower $\mathrm{p} 73$ levels, potentially limiting chemosensitiv- ity (Figure 6). In the series of 23 HNSCC cases we studied, each patient was treated preoperatively with the same cisplatin-containing chemotherapy regimen. While most patients exhibited a partial response to therapy, 2 patients showed de novo resistance and disease progression with treatment, and their tumors were among the 3 highest in miR-193a expression (not shown). While the small number of patients and the uniform responses in this series preclude definitive conclusions, this observation is consistent with our central hypothesis. Future studies in additional uniformly treated cohorts, incorporating expression of p63/p73, miR-193a and other markers, are likely to refine our ability to predict which of these patients will benefit most from chemotherapy.

While several factors may contribute to p73 upregulation in tumor cells $(42,43)$, the importance of the miR-dependent mechanism we have identified for controlling p73 levels is clear. We observe an inverse correlation between miR-193a and p73 levels in human primary HNSCC tumors, and endogenous miR inhibition alone impedes tumor growth through a p73-dependent effect. In addition to the established role for p73 as a major effector of DNA damage-induced cell death, recent data have begun to suggest that p73-dependent cell death is a key mechanism in the response to targeted inhibition of growth-factor-mediated survival pathways in tumor cells (44). Together, these findings position $\mathrm{p} 73$ as a central mediator in the response to varied environmental insults including DNA damage and growth factor withdrawal. As a result of these and other data, an intensive search is now underway to identify a means to activate 73 specifically in tumors as a therapeutic strategy (45-47). Our results uncover a new means of eliciting specific p73 upregulation through miR inhibition. This approach provides the possibility of enhancing the therapeutic effect of both chemotherapy and the future generation of targeted anticancer agents.

\section{Methods}

Tissue culture. The cell lines JHU-029 and A549 were the gift of David Sidransky (Johns Hopkins University, Baltimore, Maryland, USA). Stocks of JHU-029 used in this study express no WT p53 due to a frameshift mutation in p53 codon 108 that results in premature truncation. Primary MEFs were prepared from embryonic day $13.5 \mathrm{WT}$ and $p 53^{-/-}$littermate embryos and were immortalized by retroviral transduction of the E1A oncogene. Tetracycline-inducible HA-TAp73b cells were established in JHU-029 using the TREx plasmid system (Invitrogen). Cells were maintained at $37^{\circ} \mathrm{C}$ with $5 \% \mathrm{CO}_{2}$ in either RPMI or DMEM supplemented with $10 \% \mathrm{FBS}$, penicillin, and streptomycin. Human primary keratinocytes were prepared from newborns' foreskins as described (48) and were used at passages 2 to 4 . Human keratinocytes and OKF6 (OKF6-TERT1) (49) were cultured as described (48). Murine SCC cells were derived from primary DMBA-induced tumors (see below) and cultured in R\&G medium (50).

Murine SCC model and tumor transplant. All animals were housed and treated in accordance with protocols approved by the Subcommittee on Research Animal Care at the Massachusetts General Hospital. Murine squamous tumors were generated by treating 6-week-old mice of mixed C57BL/6 and CD1 genetic background typically over the dorsal spine with 25 mg DMBA weekly for 25 weeks. Primary murine SCC tumors were disaggregated using collagenase then FACS-sorted for viability and CD31/CD45 negativity. Sorted tumor cells $\left(5 \times 10^{4}\right.$ cells $)$ were incubated for 2 hours with either anti-miR-193* or anti-miR-Ct at $37^{\circ} \mathrm{C}, 3 \% \mathrm{O}_{2}$, $10 \% \mathrm{CO}_{2}$. Cells were then resuspended in 50\% Matrigel (BD Bioscience) and $50 \%$ PBS and were subcutaneously injected into the dorsal flanks of $\mathrm{nu} / \mathrm{nu}$ mice. The next day, mice were injected with cisplatin (Novaplus) at a concentration of $5 \mathrm{mg} / \mathrm{kg}$ via intraperitoneal injection. Animals were 
examined daily, and tumor volumes were determined by bidirectional measuring with calipers and calculated using the following formula: tumor volume $\left(\mathrm{mm}^{3}\right)=$ width $^{2} \times$ length $/ 2$. Harvested tumors were fixed in $10 \%$ formalin (Fisher Scientific) to perform histology.

Conditional $p 63 \mathrm{KO}$ mouse model. p63 flox mice (3), which harbor loxP recognition sites for the Cre recombinase flanking the genomic region encoding the DNA-binding domain of p63, were crossed with K14CreERTam mice (34). Mice were injected intraperitoneally with $75 \mathrm{mg} / \mathrm{kg}$ of tamoxifen (Sigma-Aldrich) for 5 consecutive days. Mice were sacrificed 21 days after final tamoxifen treatment.

Micro-RNA microarray analysis. For each sample, $2 \mu \mathrm{g}$ of TrizoL-extracted total RNA were purified using the RNeasy Mini Cleanup Kit (QIAGEN). After having passed sample quality control on the Bioanalyzer 2100, the samples were labeled using the miRCURY Hy3/Hy5 Power Labeling Kit and hybridized on the miRCURY LNA Array (v.10.0) (Exiqon). Spike-in controls were added in various concentrations covering the full signal range in both Hy 3 and Hy 5 labeling reactions, giving the opportunity to evaluate the labeling reaction, hybridization, and the performance of the array experiment in general. The quantified signals (background correction) were normalized using the global Lowess (LOcally WEighted Scatterplot Smoothing) regression algorithm. The diagram only represent the miRs that passed the filtering criteria across samples: fold change $>1.50$. The microarray data discussed in this publication have been deposited in NCBI Gene Expression Omnibus and are accessible through GEO Series accession number GSE25524 (http:// www.ncbi.nlm.nih.gov/geo/query/acc.cgi?acc=GSE25524).

Transient transfection of pre-miR and anti-miR. All the pre-miR and anti-miR were obtained from Ambion and transfected at a final concentration of $30 \mathrm{nM}$ using the siPORT NeoFX Transfection Agent (Ambion) according to the manufacturer's protocol. The anti-pre-miR and anti-miR negative controls used are random sequences that have been tested in human cell lines and tissues and validated to not produce identifiable effects.

Luciferase promoter and 3' UTR reporter assay. Cells were seeded in 24-well plates and were transfected with the indicated firefly luciferase constructs together with an SV40-renilla control vector. Lysates were prepared at 40 hours, and luciferase activity was measured using the Dual Luciferase Reporter Assay system (Promega) and a luminometer (MicroLumat Plus; EG\&G Berthold).

Western blot, immunoprecipitation, and ChIP. These were performed as described (8). Antibodies used against the indicated proteins are as follows: p63 (4A4; Sigma-Aldrich), polyclonal p63 (for IP, H-129; Santa Cruz Biotechnology Inc.), Drosha (D28B1; Cell Signaling), p73 (Ab-2; Calbiochem), p53 (DO1; Santa Cruz Biotechnology Inc.), HA11 (Covance), FLAG (M2; Sigma-Aldrich), and $\beta$-tubulin (Santa Cruz Biotechnology Inc.). See Supplemental Table 3 for primer sequences.

Lentiviral and retroviral production and $m R N A$ QRT-PCR. These assays were all performed as described (8). For shRNA-targeted sequences, see Supplemental Table 2. P63-directed shRNA was validated not to cross-react with p53 or p73 (8). Primer sequences for mRNA quantitative RT-PCR (QRT$\mathrm{PCR}$ ) are shown in Supplemental Table 4.

Human tumor analysis. Primary human tumor specimens with the histologic diagnosis of HNSCC were collected prior to treatment. Use of tissues and clinical data for this study were approved by the MGH/Partners Human Research Committee (Boston, Massachusetts, USA). The committee has deemed that informed consent is not required since this study involves only retrospective analysis of discarded tissue collected in the course of routine clinical care. Total RNA preparation was carried out on macrodissected tumor-enriched portions following pathology review. The $\Delta \mathrm{Np} 63$ and TAp73 quantification from tumor samples was performed using the TaqMan (Applied Biosystems) strategy according to the manufacturer's protocol. Gene expression is normalized to $\beta$-actin. Primer and probe sequences are available in Supplemental Tables 5 and 6.

miR QRT-PCR. A specific RT was performed for each miR from 100 ng of total RNA, using a specific stem-loop RT primer $(50 \mathrm{nM})$ and the MultiScribe Reverse transcriptase (Applied Biosystems). The RT conditions were as follows: 30 minutes at $16^{\circ} \mathrm{C}$ followed by 30 seconds at $20^{\circ} \mathrm{C}$, 30 seconds at $42^{\circ} \mathrm{C}, 1$ second at $50^{\circ} \mathrm{C}$ for 60 cycles, and finally 5 minutes at $85^{\circ} \mathrm{C}$. Mature miRs' expression levels were measured by real-time QRTPCR using the iQ SYBR Green Supermix reagent (Bio-Rad) and an Opticon real-time PCR detector system (MJ Research). The expression of each gene was normalized to the small nuclear U6B RNA or 5S ribosomal RNA as a reference, and relative levels were calculated from a 4-point standard curve. All experiments were performed in triplicate. Primer sequences are shown in Supplemental Tables 7 and 8.

Statistics. For each experimental data point, the SEM from replicate experiments was calculated as noted in the legends and is shown as error bars. For Figure 2D, Figure 6A, and Supplemental Figure 5C, the Pearson product-moment correlation coefficient $\left(R^{2}\right)$ was calculated, and a 2-tailed $P$ value was generated from a probability table. For in vivo experiments, 2-way repeated measured ANOVA analysis was performed to further assess the statistical significance of the cisplatin/anti-miR193* interactions, using Prism 3.0 software and based on the null hypothesis that there was no interaction between cisplatin and anti-miR-193*.

\section{Acknowledgments}

We thank Alea Mills for the gift of the $p 63^{l o x}$ mice; Peter M. Sadow for pathological review of HNSCC specimens; Philippe Hulin, Nantes University, IFR 26, PICell, for time lapse microscopy; Zachary Nash for technical assistance; and Lee Zou, Shobha Vasudevan, and members of the Ellisen laboratory for critical review of the manuscript. This work was supported by NIH RO1 DE-015945 (to L.W. Ellisen), by NIH K08 DE-020139 (to S.M. Rothenberg), and by Fondation pour la Recherche Medicale and Susan G. Komen for the Cure KG091277 (to B. Ory).

Received for publication June 1, 2010, and accepted in revised form November 10, 2010.

Address correspondence to: Leif W. Ellisen, MGH Cancer Center, GRJ-904, 55 Fruit Street, Boston, Massachusetts 02114, USA. Phone: 617.726.4315; Fax: 617.726.8623; E-mail: ellisen@helix. mgh.harvard.edu.
1. Vousden KH, Prives C. Blinded by the light: The growing complexity of p53. Cell. 2009;137(3):413-431.

2. Wang W, El-Deiry WS. Restoration of p53 to limit tumor growth. Curr Opin Oncol. 2008;20(1):90-96.

3. Keyes WM, Wu Y, Vogel H, Guo X, Lowe SW, Mills AA. p63 deficiency activates a program of cellular senescence and leads to accelerated aging. Genes Dev. 2005;19(17):1986-1999.

4. Mills AA, Zheng B, Wang XJ, Vogel H, Roop DR, Bradley A. p63 is a p53 homologue required for limb and epidermal morphogenesis. Nature. 1999; 398(6729):708-713.
5. Yang A, et al. p63 is essential for regenerative proliferation in limb, craniofacial and epithelial development. Nature. 1999;398(6729):714-718.

6. Hibi K, et al. AIS (p63) is an oncogene amplified in squamous cell carcinoma. Proc Natl Acad Sci U S A. 2000;97(10):5462-5467.

7. Thurfjell N, Coates PJ, Vojtesek B, Benham-Motlagh P, Eisold M, Nylander K. Endogenous p63 acts as a survival factor for tumour cells of SCCHN origin. Int J Mol Med. 2005;16(6):1065-1070.

8. Rocco JW, Leong CO, Kuperwasser N, DeYoung MP, Ellisen LW. p63 mediates survival in squamous cell carcinoma by suppression of p73-dependent apoptosis. Cancer Cell. 2006;9(1):45-56.

9. Tomasini R, et al. TAp73 knockout shows genomic instability with infertility and tumor suppressor functions. Genes Dev. 2008;22(19):2677-2691.

10. Wilhelm MT, et al. Isoform-specific p73 knockout mice reveal a novel role for $\{$ Delta $\} \mathrm{Np} 73$ in the DNA damage response pathway. Genes Dev. 2010; 24(6):549-560

11. Gong JG, et al. The tyrosine kinase c-Abl regulates p73 in apoptotic response to cisplatin-induced DNA damage. Nature. 1999;399(6738):806-809. 
12. Irwin MS, Kondo K, Marin MC, Cheng LS, Hahn WC, Kaelin WG Jr. Chemosensitivity linked to p73 function. Cancer Cell. 2003;3(4):403-410.

13. Urist M, Tanaka T, Poyurovsky MV, Prives C. p73 induction after DNA damage is regulated by checkpoint kinases Chk1 and Chk2. Genes Dev. 2004; 18(24):3041-3054.

14. Deyoung MP, Ellisen LW. p63 and p73 in human cancer: defining the network. Oncogene. 2007; 26(36):5169-5183.

15. Yang A, Kaghad M, Caput D, McKeon F. On the shoulders of giants: p63, p73 and the rise of p53. Trends Genet. 2002;18(2):90-95.

16. Harms K, Nozell S, Chen X. The common and distinct target genes of the p53 family transcription factors. Cell Mol Life Sci. 2004;61(7-8):822-842.

17. Coutandin D, et al. Conformational stability and activity of $\mathrm{p} 73$ require a second helix in the tetramerization domain. Cell Death Differ. 2009; 16(12):1582-1589.

18. Choi HR, Batsakis JG, Zhan F, Sturgis E, Luna MA, El-Naggar AK. Differential expression of p53 gene family members p63 and p73 in head and neck squamous tumorigenesis. Hum Pathol. 2002; 33(2):158-164.

19. DeYoung MP, Johannessen CM, Leong CO, Faquin W, Rocco JW, Ellisen LW. Tumor-specific p73 upregulation mediates p63 dependence in squamous cell carcinoma. Cancer Res. 2006;66(19):9362-9368.

20. Li Y, Peart MJ, Prives C. Stxbp4 regulates DeltaNp63 stability by suppression of RACK1-dependent degradation. Mol Cell Biol. 2009;29(14):3953-3963.

21. Li Y, Zhou Z, Chen C. WW domain-containing E3 ubiquitin protein ligase 1 targets p 63 transcription factor for ubiquitin-mediated proteasomal degradation and regulates apoptosis. Cell Death Differ. 2008;15(12):1941-1951.

22. Watson IR, Irwin MS. Ubiquitin and ubiquitinlike modifications of the p53 family. Neoplasia. 2006;8(8):655-666

23. He L, et al. A microRNA component of the p53 tumour suppressor network. Nature. 2007; 447(7148):1130-1134

24. Chang TC, et al. Transactivation of miR-34a by p53 broadly influences gene expression and promotes apoptosis. Mol Cell. 2007;26(5):745-752.

25. Rocca A, et al. Pathologic complete remission rate after cisplatin-based primary chemotherapy in breast cancer: correlation with p63 expression. Cancer Chemother Pharmacol. 2008;61(6):965-971.

26. Zangen R, Ratovitski E, Sidransky D. DeltaNp63alpha levels correlate with clinical tumor response to cisplatin. Cell Cycle. 2005;4(10):1313-1315.

27. Vester B, et al. Locked nucleoside analogues expand the potential of DNAzymes to cleave structured RNA targets. BMC Mol Biol. 2006;7:19.

28. Westfall MD, Mays DJ, Sniezek JC, Pietenpol JA The $\Delta$ Np63alpha phosphoprotein binds the $\mathrm{p} 21$ and $14-3-3$ sigma promoters in vivo and has transcriptional repressor activity that is reduced by Hay-Wells syndrome-derived mutants. Mol Cell Biol. 2003;23(7):2264-2276.

29. Betel D, Wilson M, Gabow A, Marks DS, Sander C. The microRNA.org resource: targets and expression. Nucleic Acids Res. 2008;36(Database issue):D149-D153

30. Griffiths-Jones S, Saini HK, van Dongen S, Enright AJ. miRBase: tools for microRNA genomics. Nucleic Acids Res. 2008;36(Database issue):D154-D158.

31. John B, Enright AJ, Aravin A, Tuschl T, Sander C, Marks DS. Human microRNA targets. PLoS Biol. 2004;2(11):e363.

32. Ambros V, et al. A uniform system for microRNA annotation. RNA. 2003;9(3):277-279.

33. Celli J, et al. Heterozygous germline mutations in the p53 homolog p 63 are the cause of EEC syndrome. Cell. 1999;99(2):143-153.

34. Vasioukhin V, Degenstein L, Wise B, Fuchs E. The magical touch: genome targeting in epidermal stem cells induced by tamoxifen application to mouse skin. Proc Natl Acad Sci U S A. 1999;96(15):8551-8556.

35. Leong CO, Vidnovic N, DeYoung MP, Sgroi D, Ellisen LW. The $\mathrm{p} 63 / \mathrm{p} 73$ network mediates chemosensitivity to cisplatin in a biologically defined subset of primary breast cancers. J Clin Invest. 2007; 117(5):1370-1380.

36. Bergamaschi D, et al. p53 polymorphism influences response in cancer chemotherapy via modulation of p73-dependent apoptosis. Cancer Cell. 2003; 3(4):387-402.

37. Frazer KA, Pachter L, Poliakov A, Rubin EM, Dubchak I. VISTA: computational tools for comparative genomics. Nucleic Acids Res. 2004;32(Web Server issue):W273-W279.

38. Ku TK, Nguyen DC, Karaman M, Gill P, Hacia JG, Crowe DL. Loss of p53 expression correlates with metastatic phenotype and transcriptional profile in a new mouse model of head and neck cancer. Mol Cancer Res. 2007;5(4):351-362.

39. Perrone F, et al. TP53 mutations and pathologic complete response to neoadjuvant cisplatin and fluorouracil chemotherapy in resected oral cavity squamous cell carcinoma. J Clin Oncol. 2010; 28(5):761-766.

40. Poeta ML, et al. TP53 mutations and survival in squamous-cell carcinoma of the head and neck. NEngl J Med. 2007;357(25):2552-2561.

41. Lunghi P, Costanzo A, Mazzera L, Rizzoli V, Levrero $\mathrm{M}$, Bonati A. The $\mathrm{p} 53$ family protein $\mathrm{p} 73$ provides new insights into cancer chemosensitivity and targeting. Clin Cancer Res. 2009;15(21):6495-6502.

42. Irwin $\mathrm{M}$, et al. Role for the p53 homologue p73 in E2F-1-induced apoptosis. Nature. 2000; 407(6804):645-648

43. Zaika A, Irwin M, Sansome C, Moll UM. Oncogenes induce and activate endogenous $\mathrm{p} 73$ protein. J Biol Chem. 2001;276(14):11310-11316.

44. Sun Q, et al. PUMA mediates EGFR tyrosine kinase inhibitor-induced apoptosis in head and neck cancer cells. Oncogene. 2009;28(24):2348-2357.

45. Rosenbluth JM, Mays DJ, Pino MF, Tang LJ, Pietenpol JA. A gene signature-based approach identifies mTOR as a regulator of p73. Mol Cell Biol. 2008; 28(19):5951-5964.

46. Vilgelm A, El-Rifai W, Zaika A. Therapeutic prospects for $\mathrm{p} 73$ and $\mathrm{p} 63$ : rising from the shadow of p53. Drug Resist Updat. 2008;11(4-5):152-163.

47. Wang W, Kim SH, El-Deiry WS. Small-molecule modulators of p53 family signaling and antitumor effects in p53-deficient human colon tumor xenografts. Proc Natl Acad Sci U S A. 2006;103(29):11003-11008.

48. Ellisen LW, et al. REDD1, a developmentally regulated transcriptional target of $\mathrm{p} 63$ and $\mathrm{p} 53$, links p63 to regulation of reactive oxygen species. Mol Cell. 2002;10(5):995-1005.

49. Dickson MA, et al. Human keratinocytes that express hTERT and also bypass a p16(INK4a)-enforced mechanism that limits life span become immortal yet retain normal growth and differentiation characteristics. Mol Cell Biol. 2000;20(4):1436-1447.

50. Gurumurthy S, Hezel AF, Sahin E, Berger JH, Bosenberg MW, Bardeesy N. LKB1 deficiency sensitizes mice to carcinogen-induced tumorigenesis. Cancer Res. 2008;68(1):55-63. 